\title{
Front Matter: Volume 8239
}

, "Front Matter: Volume 8239," Proc. SPIE 8239, High Power Laser Materials Processing: Lasers, Beam Delivery, Diagnostics, and Applications, 823901 (15 February 2012); doi: 10.1117/12.923569

SPIE. Event: SPIE LASE, 2012, San Francisco, California, United States 


\section{PROCEEDINGS OF SPIE}

\section{High Power Laser Materials Processing: Lasers, Beam Delivery, Diagnostics, and Applications}

\section{Eckhard Beyer}

Timothy Morris

Editors

24-26 January 2012

San Francisco, California, United States

Sponsored and Published by

SPIE 
The papers included in this volume were part of the technical conference cited on the cover and title page. Papers were selected and subject to review by the editors and conference program committee. Some conference presentations may not be available for publication. The papers published in these proceedings reflect the work and thoughts of the authors and are published herein as submitted. The publisher is not responsible for the validity of the information or for any outcomes resulting from reliance thereon.

Please use the following format to cite material from this book:

Author(s), "Title of Paper," in High Power Laser Materials Processing: Lasers, Beam Delivery, Diagnostics, and Applications, edited by Eckhard Beyer, Timothy Morris, Proceedings of SPIE Vol. 8239 (SPIE, Bellingham, WA, 2012) Article CID Number.

ISSN 0277-786X

ISBN 9780819488824

Published by

SPIE

P.O. Box 10, Bellingham, Washington 98227-0010 USA

Telephone +1 3606763290 (Pacific Time) · Fax +1 3606471445

SPIE.org

Copyright (C) 2012, Society of Photo-Optical Instrumentation Engineers

Copying of material in this book for internal or personal use, or for the internal or personal use of specific clients, beyond the fair use provisions granted by the U.S. Copyright Law is authorized by SPIE subject to payment of copying fees. The Transactional Reporting Service base fee for this volume is $\$ 18.00$ per article (or portion thereof), which should be paid directly to the Copyright Clearance Center (CCC), 222 Rosewood Drive, Danvers, MA 01923. Payment may also be made electronically through CCC Online at copyright.com. Other copying for republication, resale, advertising or promotion, or any form of systematic or multiple reproduction of any material in this book is prohibited except with permission in writing from the publisher. The CCC fee code is 0277-786X/12/\$18.00.

Printed in the United States of America.

Publication of record for individual papers is online in the SPIE Digital Library.

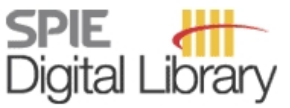

SPIEDigitalLibrary.org

Paper Numbering: Proceedings of SPIE follow an e-First publication model, with papers published first online and then in print and on CD-ROM. Papers are published as they are submitted and meet publication criteria. A unique, consistent, permanent citation identifier (CID) number is assigned to each article at the time of the first publication. Utilization of CIDs allows articles to be fully citable as soon as they are published online, and connects the same identifier to all online, print, and electronic versions of the publication. SPIE uses a six-digit CID article numbering system in which:

- The first four digits correspond to the SPIE volume number.

- The last two digits indicate publication order within the volume using a Base 36 numbering system employing both numerals and letters. These two-number sets start with 00, 01, 02, 03, 04, $05,06,07,08,09,0 A, 0 B \ldots 0 Z$, followed by 10-1Z, 20-2Z, etc.

The CID number appears on each page of the manuscript. The complete citation is used on the first page, and an abbreviated version on subsequent pages. Numbers in the index correspond to the last two digits of the six-digit CID number. 


\section{Contents}

vii Conference Committee

\section{SESSION 1 INVITED SESSION}

823903 The story of laser brazing technology (Invited Paper) [8239-02]

P. Hoffmann, R. Dierken, ERLAS Erlanger Lasertechnik GmbH (Germany)

823904 Innovations in laser cladding and direct metal deposition (Invited Paper) [8239-03]

F. Brückner, S. Nowotny, Fraunhofer IWS (Germany); C. Leyens, Fraunhofer IWS (Germany) and Technische Univ. Dresden (Germany)

823905 Structural strengthening of rocket nozzle extension by means of laser metal deposition (Invited Paper) [8239-04]

M. Honoré, Force Institute (Denmark); L. Brox, M. Hallberg, Volvo Aero Corp. (Sweden)

\section{SESSION 2 LASERS AND LASER SYSTEMS IN MACRO PROCESSING}

823907 Frequency doubled high-power disk lasers in pulsed and continuous-wave operation [8239-06]

S. Weiler, TRUMPF Inc. (United States); A. Hangst, C. Stolzenburg, I. Zawischa, D. Sutter, A. Killi, S. Kalfhues, TRUMPF Laser GmbH \& Co. KG (Germany); U. Kriegshaeuser, M. Holzer, TRUMPF Laser- und Systemtechnik GmbH (Germany); D. Havrilla, TRUMPF Inc. (United States)

823908 Lasers for industrial production processing: tailored tools with increasing flexibility [8239-07] W. Rath, ROFIN-SINAR Laser GmbH (Germany)

8239 OA High-power transmission characterization of Chalcogenide glasses using a Tm:fiber laser system [8239-09]

P. Kadwani, J. Bradford, R. A. Sims, CREOL, The College of Optics and Photonics, Univ. of Central Florida (United States); D. Musgraves, K. Richardson, Clemson Univ. (United States); L. Shah, M. C. Richardson, CREOL, The College of Optics and Photonics, Univ. of Central Florida (United States)

\section{SESSION $3 \quad$ APPLICATIONS: WELDING}

$8239 \mathrm{OB}$ Advantages of fibre lasers in 3D metal cutting and welding applications supported by a 'beam in motion (BIM)' beam delivery system [8239-10]

T. Scheller, A. Bastick, M. Griebel, JENOPTIK Automatisierungstechnik GmbH (Germany)

8239 OC T-joints of Ti alloys with hybrid laser-MIG welding: macro-graphic and micro-hardness analyses [8239-12]

R. Spina, D. Sorgente, G. Palumbo, L. D. Scintilla, Politecnico di Bari (Italy); M. Brandizzi,

A. A. Satriano, Consorzio CALEF (Italy); L. Tricarico, Politecnico di Bari (Italy) 
8239 OD Improvements of the welding performance of plasma arcs by a superimposed fibre laser beam [8239-13]

A. Mahrle, S. Rose, M. Schnick, T. Pinder, Technische Univ. Dresden (Germany); E. Beyer, Technische Univ. Dresden (Germany) and Fraunhofer IWS Dresden (Germany); U. Füssel, Technische Univ. Dresden (Germany)

8239 OE Bead characterization of disk-laser butt welding of thin AA 2024 sheets [8239-41]

F. Caiazzo, V. Alfieri, F. Cardaropoli, V. Sergi, Univ. degli Studi di Salerno (Italy)

8239 OF Through the optical combiner monitoring in remote fiber laser welding of zinc coated steels [8239-38]

D. Colombo, B. M. Colosimo, B. Previtali, Politecnico di Milano (Italy); D. Bassan, M. Lai, Ctr. Ricerche Fiat S.C.p.A. (Italy); G. Masotti, El.En. S.p.A. (Italy)

\section{SESSION 4 APPLICATIONS: SURFACE TREATMENT AND CLADDING}

8239 OG Identification of phase transformation using optical emission spectroscopy for direct metal deposition process (Invited Paper) [8239-15]

L. Song, Univ. of Michigan (United States); C. Wang, Univ. of Michigan (United States) and Dalian Univ. of Technology (China); J. Mazumder, Univ. of Michigan (United States)

$8239 \mathrm{OH}$ The use of novel, direct diode lasers for large area hard-facing and high deposition rate cladding to enhance surface wear and corrosion resistance [8239-16]

S. Brookshier, J. Washko, K. Parker, F. Gaebler, W. Juchmann, Coherent, Inc. (United States)

8239 Ol Laser heat treatment with latest system components [8239-17]

S. Bonss, J. Hannweber, U. Karsunke, S. Kuehn, M. Seifert, Fraunhofer IWS Dresden (Germany); E. Beyer, Fraunhofer IWS Dresden (Germany) and Technische Univ. Dresden (Germany)

$8239 \mathrm{OJ} \quad$ Local heat treatment of high strength steels with zoom-optics and 10kW-diode laser [8239-18]

M. Baumann, V. Krause, Laserline GmbH (Germany); G. Bergweiler, Fraunhofer-Institut für Lasertechnik (Germany); M. Flaischerowitz, F+K Werkstoffprüfung und Labor GmbH (Germany); J. Banik, ThyssenKrupp Steel Europe AG (Germany)

$8239 \mathrm{OL} \quad$ Multi-kW laser cladding using cylindrical collimators and square-formed fibers [8239-20] M. Blomqvist, S. Campbell, Optoskand AB (Sweden); J. Latokartano, J. Tuominen, Tampere Univ. of Technology (Finland)

\section{SESSION 5 APPLICATIONS: CUTTING}

8239 OM Wavelength dependency in high power laser cutting and welding (Invited Paper) [8239-21] D. Havrilla, S. Ziermann, TRUMPF Inc. (United States); M. Holzer, TRUMPF Laser- und Systemtechnik GmbH (Germany)

$8239 \mathrm{ON} \quad$ Energy balance in disk and $\mathrm{CO}_{2}$ laser beam inert gas fusion cutting [8239-22] L. D. Scintilla, L. Tricarico, Politecnico di Bari (Italy); A. Wetzig, Fraunhofer IWS Dresden (Germany); E. Beyer, Fraunhofer IWS Dresden (Germany) and Univ. of Technology Dresden (Germany) 
823900 Applicability of various beam sources for high power laser cutting of non-oriented electrical steel [8239-23]

R. Siebert, Fraunhofer IWS Dresden (Germany) and TRUMPF Sachsen GmbH (Germany) and Technische Univ. Dresden (Germany); H. Thonig, TRUMPF Sachsen GmbH (Germany);

A. Wetzig, Fraunhofer IWS Dresden (Germany); E. Beyer, Fraunhofer IWS Dresden (Germany) and Technische Univ. Dresden (Germany)

8239 OP Tailor cutting of crystalline solar cells by laser micro jet [8239-24]

F. Bruckert, Synova S.A. (Switzerland); E. Pilat, Institut National de l'Energie Solaire (France); P. Piron, P. Torres, B. Carron, B. Richerzhagen, Synova S.A. (Switzerland); M. Pirot, R. Monna, Institut National de l'Energie Solaire (France)

$82390 \mathrm{OQ}$ Combining remote ablation cutting and remote welding: opportunities and application areas [8239-25]

J. Musiol, Institute for Machine Tools and Industrial Management (Germany); M. Luetke, Fraunhofer IWS Dresden (Germany); M. Schweier, J. Hatwig, Institute for Machine Tools and Industrial Management (Germany); A. Wetzig, E. Beyer, Fraunhofer IWS Dresden (Germany); M. F. Zaeh, Institute for Machine Tools and Industrial Management (Germany)

\section{SESSION $6 \quad$ BEAM DELIVERY AND DIAGNOSTICS I}

8239 OR Mid-infrared imaging Fourier transform spectrometry for high power fiber laser irradiated fiberglass composites (Invited Paper) [8239-26]

R. I. Acosta, K. C. Gross, G. P. Perram, Air Force Institute of Technology (United States)

8239 OS Spectroscopic closed loop control of penetration depth in laser beam welding process [8239-27]

T. Sibillano, A. Ancona, CNR-IFN UOS Bari (Italy); D. Rizzi, F. Mezzapesa, Univ. degli Studi e Politecnico di Bari (Italy); A. R. Konuk, R. Aarts, B. Huis in '† Veld, Univ. Twente (Netherlands); P. M. Lugarà, CNR-IFN UOS Bari (Italy) and Univ. degli Studi e Politecnico di Bari (Italy)

8239 OT NIR-camera-based online diagnostics of laser beam welding processes [8239-28] F. Dorsch, H. Braun, S. Keßler, D. Pfitzner, TRUMPF Werkzeugmaschinen GmbH + Co. KG (Germany); V. Rominger, TRUMPF Laser- und Systemtechnik GmbH (Germany)

8239 OU High-power fiber optic cable with integrated active sensors for live process monitoring [8239-29]

O. Blomster, M. Blomqvist, H. Bergstrand, M. Pålsson, Optoskand AB (Sweden)

\section{SESSION 7 BEAM DELIVERY AND DIAGNOSTICS II}

8239 OV Beam delivery systems and processing heads for $1 \mu \mathrm{m}$ high brightness laser cutting systems [8239-30]

H. Zimer, R. Niedrig, B. Wedel, HIGHYAG Lasertechnologie GmbH (Germany)

8239 OW Unique beam delivery and processing tools for high power solid state laser processing [8239-31]

T. Ryba, D. Havrilla, TRUMPF Inc. (United States); M. Holzer, M. Bea, TRUMPF Laser- und

Systemtechnik GmbH (Germany) 
8239 0X Maximum uptime and minimum focus shift in high-power $1 \mu \mathrm{m}$ laser beam delivery [8239-32]

T. Kugler, Laser Mechanisms, Inc. (United States)

8239 oY Ultra-low absorption glasses and optical coatings for reduced thermal focus shift in high power optics [8239-33]

D. T. Carpenter, C. S. Wood, O. Lyngnes, N. G. Traggis, Precision Photonics Corp. (United States)

$8239 \mathrm{OZ}$ Self-compensation of thermal lensing in optics for high-brightness solid-state lasers [8239-34]

S. Piehler, C. Thiel, A. Voss, M. Abdou Ahmed, T. Graf, Univ. Stuttgart (Germany)

823910 Fabrication and characteristic of a multicore fiber for high power laser delivery [8239-35] H. Wei, S. Chen, J. Li, C. Tang, Yangtze Optical Fibre and Cable Co., Ltd. (China); P. Yan, Shenzhen Univ. (China)

\section{POSTER SESSION}

823911 Controlling the thermally induced focal shift in laser processing heads [8239-36] J.-P. Negel, F. Abt, D. Blázquez-Sánchez, A. Austerschulte, M. Hafner, T. Liebig, P. von Strobl-Albeg, R. Weber, M. Abdou Ahmed, A. Voss, T. Graf, Univ. Stuttgart (Germany)

823913 Study of a fiber laser assisted friction stir welding process [8239-39]

G. Casalino, S. Campanelli, A. D. Ludovico, N. Contuzzi, A. Angelastro, Politecnico di Bari (Italy)

823914 Parameters in selective laser melting for processing metallic powders [8239-40]

T. Kurzynowski, E. Chlebus, B. Kuźnicka, J. Reiner, Wroclaw Univ. of Technology (Poland)

Author Index 


\title{
Conference Committee
}

\author{
Symposium Chairs
}

Friedhelm Dorsch, TRUMPF Werkzeugmaschinen GmbH + Co. KG (Germany)

Alberto Piqué, Naval Research Laboratory (United States)

Symposium Cochairs

Bo Gu, IPG Photonics Corporation (China)

Andreas Tünnermann, Friedrich-Schiller-Universität Jena (Germany)

Program Track Chair

Gregory J. Quarles, BE Meyers \& Company Inc. (United States)

Conference Chairs

Eckhard Beyer, Fraunhofer-Institut für Werkstoff- und Strahltechnik (Germany)

Timothy Morris, TRUMPF Inc. (United States)

Program Committee

Milan Brandt, RMIT University (Australia)

Craig Bratt, Fraunhofer USA, Inc. (United States)

Edward Chlebus, Wroclaw University of Technology (Poland)

Ingomar Kelbassa, RWTH Aachen (Germany)

Wolfgang Knapp, Coopération Laser Franco-Allemande (France)

Isamu Miyamoto, Osaka University (Japan)

Thomas P. Pearsall, European Photonics Industry Consortium (France)

Silke Pflueger, Laserline Inc. (United States)

Michael Schmidt, Friedrich-Alexander-Universität Erlangen-Nürnberg (Germany)

Jens Standfuss, Fraunhofer-Institut für Werkstoff- und Strahltechnik (Germany)

Anja Techel, Fraunhofer-Institut für Werkstoff- und Strahltechnik (Germany)

Luigi Tricarico, Politecnico di Bari (Italy)

Kunihiko Washio, Paradigm Laser Research Ltd. (Japan)

Andreas Wetzig, Fraunhofer-Institut für Werkstoff- und Strahltechnik (Germany)

Minlin Zhong, Tsinghua University (China) 
Session Chairs

1 Invited Session

Eckhard Beyer, Fraunhofer-Institut für Werkstoff- und Strahltechnik (Germany)

$2 \quad$ Lasers and Laser Systems in Macro Processing

Silke Pflueger, Laserline Inc. (United States)

3 Applications: Welding

Timothy Morris, TRUMPF Inc. (United States)

$4 \quad$ Applications: Surface Treatment and Cladding

Christoph Leyens, Technische Universität Dresden (Germany)

5 Applications: Cutting

Andreas Wetzig, Fraunhofer-Institut für Werkstoff- und Strahltechnik (Germany)

6 Beam Delivery and Diagnostics I

Kunihiko Washio, Paradigm Laser Research Ltd. (Japan)

7 Beam Delivery and Diagnostics II

Wolfgang Knapp, Coopération Laser Franco-Allemande (France) 